\title{
The Embedded Local Monitor Board upgrade proposals
}

\section{H. Binderup ${ }^{a}$, H. Boterenbrood ${ }^{b}$, P. Farthouat ${ }^{c}$, K. Nicpon ${ }^{\star c}$, P. Nikiel ${ }^{c}$, V. Ryjov', S. Schlenker ${ }^{\mathrm{C}}$}

${ }^{a}$ Aarhus University

Inge Lehmanns Gade 10, 8000, Aarhus, Denmark

${ }^{b}$ Nikhef

Science Park 105, 1098 XG, Amsterdam, The Netherlands

${ }^{c}$ CERN

CH-1211 Geneva 23, Switzerland

E-mail: kamil.nicponecern.ch

The Embedded Local Monitor Board (ELMB) is a microcontroller based plug-in module using the CANopen communication protocol. It has been widely used in LHC systems and experiments for slow-control and monitoring purposes, providing multiple, galvanically isolated analog inputs, and multiple digital inputs and outputs. While the module showed excellent performance in the past 15 years, a new design is deemed necessary due to the obsolescence of some components of the existing ELMB and the higher radiation tolerance required for the planned HL-LHC upgrade. Three development paths, one fully backward compatible and two conceptually different solutions with different levels of radiation tolerance are presented.

\section{${ }^{*}$ Speaker}




\section{Introduction}

The Embedded Local Monitor Board (ELMB)[1] is a general purpose plug-in board with 64 analog inputs, up to 16 digital inputs and up to 16 digital outputs, developed for slow-control and monitoring purposes of the LHC detector front-end electronics. It communicates via CAN bus using the CANopen protocol, one of the serial-bus based protocols supported by CERN. The board is compact $(50 \times 67 \mathrm{~mm})$ and low-power $(<0.5 \mathrm{~W})$. Figure 1 presents the block diagram of the ELMB.

While the ELMB has performed well over the years, a replacement is deemed necessary for a number of reasons. Some components have become obsolete, making it impossible to produce additional modules. The ELMB has been qualified as suitable for a total ionizing dose (TID) up to $140 \mathrm{~Gy}$, and neutron fluency of $5 \mathrm{E} 12 \mathrm{MeV}$ neutrons $/ \mathrm{cm}^{2}$, but with the planned High Luminosity upgrade of the LHC, the requirements for radiation tolerance will be much higher. Furthermore, feedback from users suggests that some additional functionality and an increased throughput would be desirable.

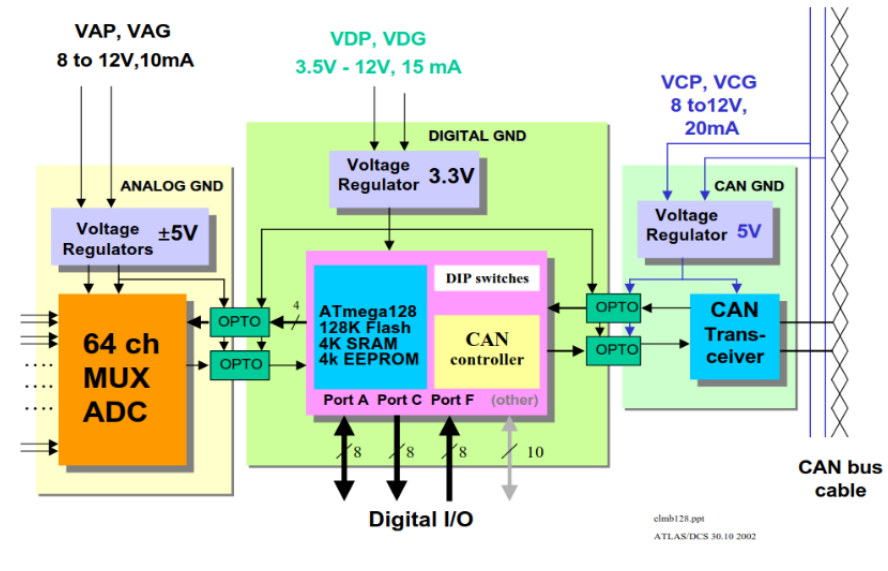

Figure 1: Architecture of the ELMB

\subsection{Development paths}

The ELMB is widely used in LHC experiments, and close to 20000 pieces were produced. For this reason it would be very advantageous to design a module that is fully hardware and software backward compatible. Unfortunately, after investigation of different solutions it was found impossible to produce a module that would also meet the requirements in terms of radiation tolerance set by the sub-detector teams. It was then decided that the best solution for users would be to have three different development paths, each focused on a different aspect of the requirements.

The first design, called ELMB2, is backward compatible with the current ELMB.

The second design, called ELMB++ GBT-SCA, based on radiation hard ASICs is aimed to satisfy the highest requirements with respect to radiation.

And a third design, called ELMB++ FPGA, is composed only of commercial off-the-shelf (COTS) components and would have the highest data throughput.

\section{Backward compatible system: ELMB2}

The backward compatible solution allowing to preserve the existing infrastructure is a very favourable one. The radiation tests of ELMB provided information about its weak points[2] and these have been improved upon for the ELMB2. By using CERN's experience with radiation testing of COTS, the ELMB design has been updated as follows:

- the now obsolete CAN controller chip has been replaced by an equivalent component;

- the voltage regulators have been replaced by more radiation tolerant ones; 
- the optocouplers, sensitive to neutron displacement damage and known as the weakest component of the ELMB design, have been replaced by digital isolators;

- the microcontroller has been replaced by a space-grade version of the same type.

In addition, the PCB was designed such that a different (non space-grade and thus cheaper) version of microcontroller of the same family, with integrated CAN controller, can be mounted instead.

\subsection{Radiation tests of ELMB2}

Twelve ELMB2 boards have been irradiated in the CHARM facility at CERN. Conditions differed from those during ELMB radiation tests[2], so a number of ELMBs were irradiated as well, for comparison. The CHARM facility provides a radiation environment[3], with conditions similar to the real operation environment in LHC detectors. The test bench was prepared in the following way:

- the current consumption of each section of each board was monitored separately;

- the standard firmware was extended with an automatic periodic check for bit flips in unused sections of RAM and EEPROM memory as well as a number of ADC, CAN and microcontroller registers and a check of the CRC of the firmware itself in flash memory;

- analog inputs were connected to voltage references to check the performance of the analog multiplexers and ADC in a radiation environment.

\subsubsection{Results of Total Ionizing Dose test}

Increase of power consumption is a good indicator of damage caused by radiation to electronics. The plots in Figure 2 compare ELMB and ELMB2 in terms of current consumption. The curves cover the range where the ELMB or ELMB2 was working correctly. The plots are showing the measurements from only one ELMB and one ELMB2, but the other boards under test showed very similar behaviour.
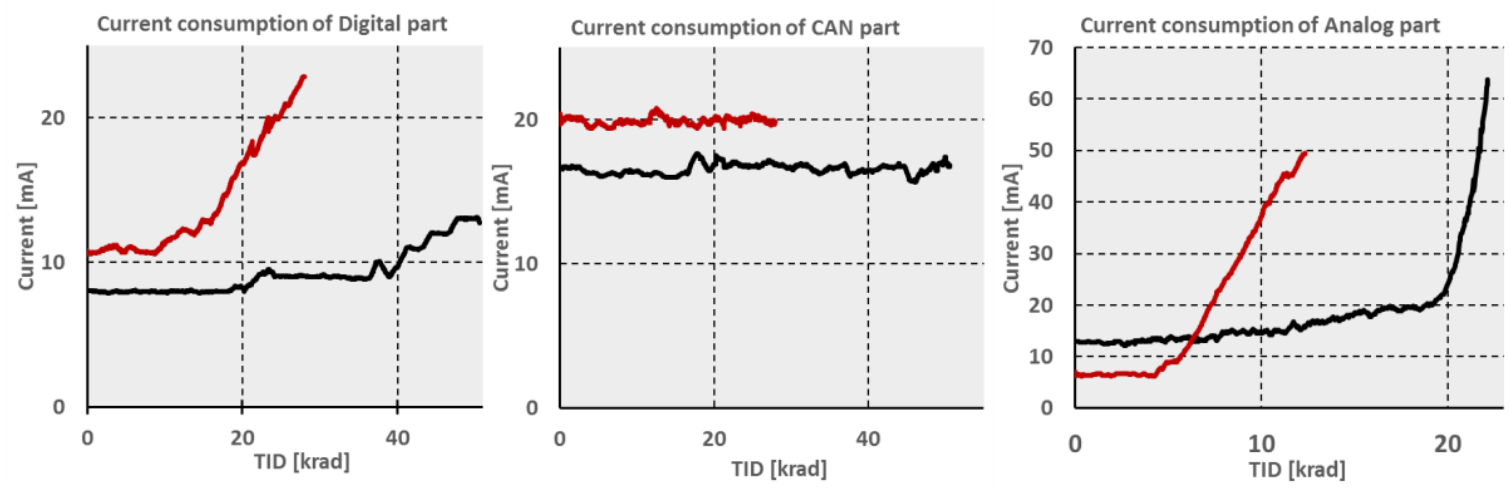

Figure 2: Current consumption of each section of ELMB over TID measured for ELMB (red) and ELMB2 (black)

This shows that the radiation tolerance has been significantly increased by the update of the ELMB design. The ADC is the most sensitive component of the ELMB2 for the total ionizing dose, which failed on most of the boards after about 22-26krad. 


\subsubsection{Analysis of Single Event Effects test}

Every 10 seconds bit flips in memories and registers detected by the automatic periodic check were reported in CAN messages during the radiation test. From these a cross-section could be estimated for each type of register or memory cell, presented in Table 1.

In addition the functional state of the board was monitored during the test. In normal operation there are some watchdogbased mechanisms implemented in the firmware allowing the module to selfrecover from misbehaviour. In some cases, an external action such as sending a reset command or performing a power cycle was required to recover functionality. A summary

\begin{tabular}{|l|c|c|c|c|}
\hline $\begin{array}{c}\text { Tested reg- } \\
\text { isters }\end{array}$ & $\begin{array}{c}\text { Number of } \\
\text { tested bits }\end{array}$ & $\begin{array}{c}\text { Number of } \\
\text { SEUs }\end{array}$ & $\begin{array}{c}\text { Fluence } \\
{\left[\mathbf{H E H} / \mathbf{c m}^{2}\right]}\end{array}$ & $\begin{array}{c}\text { Cross-section } \\
\left.\text { [cm }^{2} / \mathbf{b i t}\right]\end{array}$ \\
\hline RAM & 16384 & 2652 & $1.94 \times 10^{12}$ & $8.34 \times 10^{-14}$ \\
\hline EEPROM & 16384 & $<1$ & $1.94 \times 10^{12}$ & $<3.14 \times 10^{-17}$ \\
\hline FLASH & 327680 & $<1$ & $1.94 \times 10^{12}$ & $<1.57 \times 10^{-18}$ \\
\hline MCU reg & 80 & 3 & $1.94 \times 10^{12}$ & $1.93 \times 10^{-14}$ \\
\hline CAN reg & 272 & 44 & $1.94 \times 10^{12}$ & $8.34 \times 10^{-14}$ \\
\hline ADC reg & 288 & 2 & $9.87 \times 10^{11}$ & $3.58 \times 10^{-15}$ \\
\hline
\end{tabular}

Table 1: Estimated cross-section

\begin{tabular}{|c|c|c|}
\hline Recovery type & $\begin{array}{l}\text { functional SEEs for } \\
1.94 \times 10^{12} \mathrm{HEH} / \mathrm{cm}^{2}\end{array}$ & $\begin{array}{l}\left.\text { Average [HEH } / \mathrm{cm}^{2}\right] \\
\text { fluence for } 1 \text { error }\end{array}$ \\
\hline Power cycling & 3 & $6.47 \times 10^{11}$ \\
\hline Software reset & 2 & $9.7 \times 10^{11}$ \\
\hline Automatic & 18 & $1.08 \times 10^{11}$ \\
\hline Total & 23 & $8.43 \times 10^{10}$ \\
\hline
\end{tabular}

Table 2: Recovery of functional SEES of functional single event effects is presented in Table 2. As in the TID analysis, the values presented above were calculated for one representative ELMB2 unit. The other ELMB2 modules in the test displayed very similar behaviour.

\section{GBT-SCA-based system}

The GBT-SCA ASIC[4] is part of the GBT chip set[5] and provides an extension of the control data path to the front-end, in the form of several configurable interfaces (digital $\mathrm{I} / \mathrm{O}, \mathrm{I}^{2} \mathrm{C}$, SPI and JTAG), as well as a number of analog inputs and outputs. The GBT-SCA fulfils all required functionalities of an ELMB++. For a similar number of analog inputs as the ELMB has, two GBT-SCA chips are needed. Two different topologies based on GBT-SCA and GBT chip set may be applied. In a point-to-point topology each satellite consists of two GBT-SCA chips and one GBTx chip. In a star topology, a GBTx would be used as a hub for up to 20 ELMB++ satellites, and each satellite would consist only of GBT-SCA chips. The GBT-SCA has been designed to work with GBTx on the same ground reference potential. Unfortunately, sharing the same ground potential by satellites is not acceptable for many of the current ELMB applications, so the relevance of a star topology is limited. An example of possible topology of GBT-SCA based system is shown in Figure 3.

This system has the advantage that the GBT chip set is radiation hard. The main disadvantage is its point-to-point topology, which leads to high infrastructure costs and has an exclusively local powering scheme.

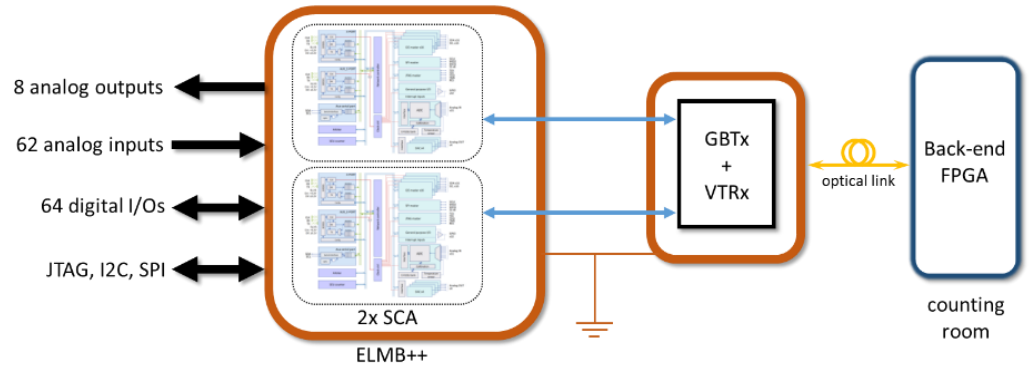

Figure 3: Topology of GBT-SCA-based system 


\section{FPGA-based system}

A third proposal for ELMB replacement is a board built with COTS components, based on an FPGA as shown in Figure 4. CERN's experience with testing and operating COTS components in radiation areas shows that with carefully selected components the requirements of radiation tolerance for most ELMB locations may be met.

Usage of discrete peripheral components like ADCs or DACs offers the possibility of galvanic separation of input/output interfaces from the communication part of the board, allowing to separately power the communication and interface parts of the module, as in the ELMB design. Moreover, as the board is based on an FPGA, the firmware

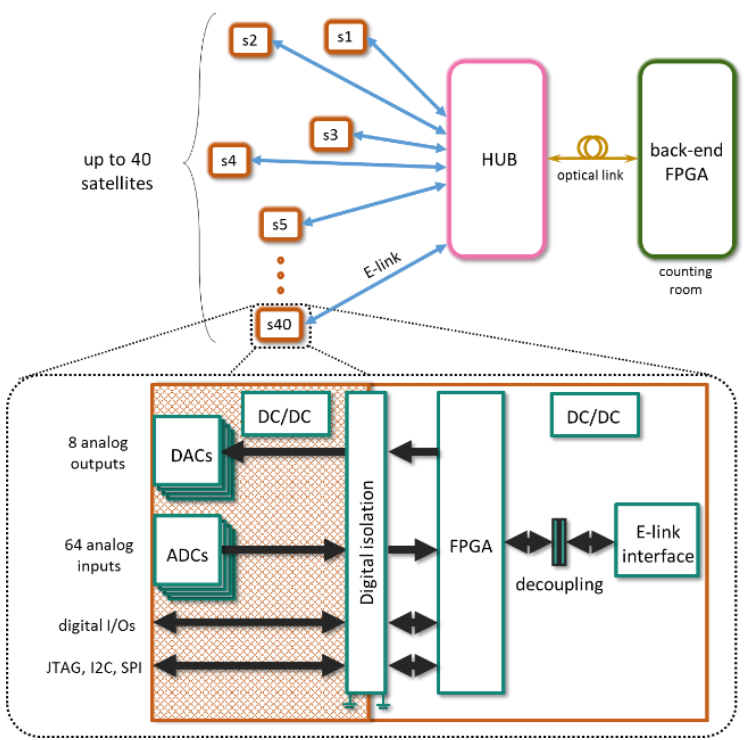

Figure 4: Topology of FPGA-based system can be tailored to user-specific needs. The proposed board will be connected in a star topology with a hub, which is based on GBTx or FPGA. In the latter case, there is the possibility to add data pre-processing logic to the hub. Each hub could serve up to 40 satellites. The hub can be placed at a distance of up to 20-30 m from the satellites, which means the radiation requirements for the hub can be lower than those for satellites.

\section{Conclusions}

Different solutions for an ELMB successor have been studied. Since it is not feasible to have a solution that is both backward compatible and meets the new radiation requirements, three different development paths are being explored. The ELMB2, a backward compatible replacement proposal has been prototyped and tested in a radiation environment. Significant gain in terms of radiation tolerance with respect to the current ELMB has been achieved, although more specific tests will need to be performed.

\section{References}

[1] B. Hallgren et al., The Embedded Local Monitor Board (ELMB) in the LHC Front-end I/O Control System, Stockholm 2001, Electronics for LHC experiments 325-330

[2] H. Boterenbrood and B. Hallgren, 2004, SEE and TID Qualification of the ELMB128 Series Production, ATL-DQ-EN-0033, EDMS ID 811704, https://edms.cern.ch/document/811704/1

[3] J. Mekki et al., CHARM: A Mixed Field Facility at CERN for Radiation Tests in Ground, Atmospheric, Space and Accelerator Representative Environments, IEEE Trans. Nucl. Sci., vol. 63, no. 4, pp. 2106-2114, 2016

[4] A. Caratelli et al., The GBT-SCA, a radiation tolerant ASIC for detector control and monitoring applications in HEP experiments, JINST $10 \mathrm{C} 03034$

[5] P. Moreira et al., The GBT Project, in proceedings of Topical Workshop on Electronics for Particle Physics, TWEPP 2009, Paris, France, 21-25 September 2009, pp. 342-346 (CERN-2009-006) 